\author{
Marquette University \\ e-Publications@Marquette
}

$9-23-2021$

\title{
On-Site/In Situ Continuous Detecting ppb-Level Metal lons in Drinking Water Using Block Loop-Gap Resonators and Machine Learning
}

\author{
Sangmin Oh \\ Marquette University \\ Imtiaz Hossen \\ Marquette University \\ Juan R. Luglio \\ JRL Engineering \\ Gusphyl Justin \\ A.O. Smith Corporate Technology Center \\ James Richie \\ Marquette University, james.richie@marquette.edu
}

See next page for additional authors

Follow this and additional works at: https://epublications.marquette.edu/electric_fac

Part of the Computer Engineering Commons, and the Electrical and Computer Engineering Commons

\section{Recommended Citation}

Oh, Sangmin; Hossen, Imtiaz; Luglio, Juan R.; Justin, Gusphyl; Richie, James; Medeiros, Henry; and Lee, Chung-Hoon, "On-Site/In Situ Continuous Detecting ppb-Level Metal lons in Drinking Water Using Block Loop-Gap Resonators and Machine Learning" (2021). Electrical and Computer Engineering Faculty Research and Publications. 675.

https://epublications.marquette.edu/electric_fac/675 


\section{Authors}

Sangmin Oh, Imtiaz Hossen, Juan R. Luglio, Gusphyl Justin, James Richie, Henry Medeiros, and ChungHoon Lee 
Marquette University

e-Publications@Marquette

\section{Electrical and Computer Engineering Faculty Research and Publications/College of Engineering}

This paper is NOT THE PUBLISHED VERSION.

Access the published version via the link in the citation below.

IEEE Transactions on Instrumentation and Measurement, Vol. 70 (September 23, 2021): 9513909. DOI. This article is (C) Institute of Electrical and Electronics Engineers and permission has been granted for this version to appear in e-Publications@Marquette. Institute of Electrical and Electronics Engineers does not grant permission for this article to be further copied/distributed or hosted elsewhere without express permission from Institute of Electrical and Electronics Engineers.

\section{On-Site/In Situ Continuous Detecting ppb- Level Metal Ions in Drinking Water Using Block Loop-Gap Resonators and Machine Learning}

Sangmin Oh

Marquette University, Milwaukee, WI

Imtiaz Hossen

Marquette University, Milwaukee, WI

Juan Luglio

JRL Engineering, Milwaukee, WI

Gusphyl Justin

A.O. Smith Corporate Technology Center, Milwaukee, WI

James E. Richie

Marquette University, Milwaukee, WI 


\section{Henry Medeiros}

Marquette University, Milwaukee, WI

Chung Hoon Lee

Marquette University, Milwaukee, WI

\section{Abstract:}

Microwave measurements and machine learning algorithms are presented to estimate metal ion concentrations in drinking water. A novel block loop gap resonator (BLGR) as a microwave probe is designed and fabricated to estimate $\mathrm{Pb}$ ion concentrations in city water as low as $1 \mathrm{ppb}$ with an rms error of $0.18 \mathrm{ppb}$. No physical contact between the BLGR probe and the water sample allows on-site/in situ continuous detection of ppb-level metal ion concentrations. The $S_{11}$ raw data (amplitude and phase) from the BLGR are used to classify and estimate metal ion concentrations using a support vector regression algorithm. The performance of the proposed method to estimate $\mathrm{Pb}$ concentrations in the presence of interfering metal ions $\left(\mathrm{Cu}^{2+}, \mathrm{Fe}^{3+}\right.$, and $\left.\mathrm{Zn}^{2+}\right)$ is also evaluated, and it is found that the average measurement error remains less than $13 \%$.

\section{SECTION I. Introduction}

Metal ions in water have a great impact on biological and environmental processes [1], [2]. While metal ions, such as sodium ( $\mathrm{Na})$, iron (Fe), copper ( $\mathrm{Cu}$ ), and zinc ( $\mathrm{Zn})$, are essential for different biochemical processes, metal ions, such as lead $(\mathrm{Pb})$, mercury $(\mathrm{Hg})$, and cadmium $(\mathrm{Cd})$, are severely toxic even at low concentrations [3].

There are several well-established technologies used to identify the concentration and type of metal ions in water. They are inductive coupled plasma mass spectrometry [4], [5], atomic absorption spectrometry [6], [7], electrochemical analysis [8], [9], fluorescence spectrometry [10], [11], and optical emission spectrometry [12], [13]. These technologies have high accuracy and sensitivity. However, they require sophisticated instruments and involve complex data analysis in a controlled environment. Hence, measurements by such systems are ex situ since they must be performed in a laboratory [14]. Most on-site/in situ sensing technologies are based on electrochemical or optical methods [9], [15]-[16][17]. Electrochemical sensors require the modification of the sensing electrode surface, whereas optical sensors require molecular markers. Both electrochemical and optical sensors are unsuitable for continuously monitoring metal ion contaminants in drinking water. Microwave resonators have been used for sensing glucose and mixed phosphate/nitrate ppm level concentrations in water [18]-[19][20].

In this article, a microwave block loop gap resonator (BLGR) is presented as a metal ion sensor. The BLGR continuously measures the concentrations of metal ions in drinking water systems. Because liquid samples of interest are placed in a glass tube inside the resonator as shown in Fig. 1(d), the BLGR has no physical contact with the sample ensuring on-site/in situ continuous monitoring of metal ions in water. Experimental results demonstrate that the BLGR is able to classify solutions of different ions $\left(\mathrm{Na}^{+}, \mathrm{K}^{+}\right.$, and $\left.\mathrm{Mg}^{2+}\right)$ in deionized (DI) water and to measure $\mathrm{Pb}$ ion concentration with $1 \mathrm{ppb}$ resolution in city water. 


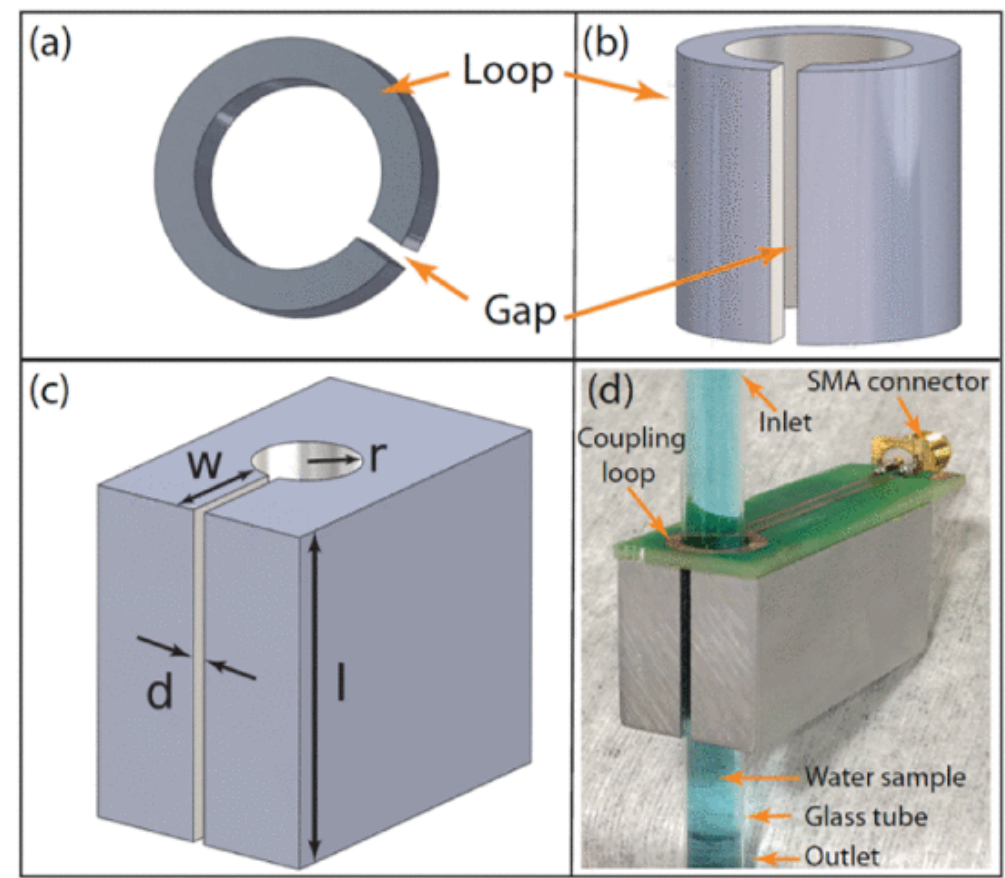

Fig. 1. Schematic of different resonators: (a) split ring resonator, (b) slotted tube cavity resonator, (c) BLGR with dimensions, and (d) integration of a BLGR, coupling loop, and water sample for the $S_{11}$ measurement. A 6.3-mm OD glass tube is placed in the BLGR loop. A water sample is introduced in the glass capillary. There is no physical contact between the BLGR and the water sample. In the picture, the water sample is colored with a blue dye to facilitate visualization.

\section{SECTION II. BLGR Sensors}

A loop gap resonator (LGR) is a microwave device consisting of a hollow cylindrical loop with a gap at its periphery. It is also known as a slotted tube cavity [21] or a split ring resonator [22]. The device can be considered as a lumped LC circuit where the loop acts as an inductor and the gap functions as a capacitor. In the presence of an electromagnetic (EM) signal, a magnetic field is induced around the loop. The gap holds electric charges that create a uniform electric field [23]. When the stored magnetic energy in the loop and the stored electric energy in the gap are equal, the LGR structure becomes resonant. An LGR is a reusable passive device with large filling factor, medium $Q$ value, and nearly uniform magnetic field in the loop [24]. It is much less susceptible to stray environmental signals while sensing than the cavity resonator. The sensor is cost-effective, as mass production is possible. LGRs are extensively used to measure different electrodynamic properties of solids. In electron spin resonance spectroscopy, a single-loop multiple-gap LGR has been introduced for the frequency range 1-10 $\mathrm{GHz}$ [25], [26]. A single-loop LGR can exhibit unwanted radiation at higher frequencies. To reduce the radiation loss, a multiloop multigap resonator, which better confines magnetic flux, has been used Wood et al. [27]. Each additional gap adds one more capacitor in series in the equivalent circuit, enabling ESR spectroscopy at a higher frequency.

As shown in Fig. 1(a) and (b), most LGR structures are cylindrical, where the loop acts as an inductor, and a parallel slot is cut from the body to create a capacitor. The capacitance of the cylindrical LGR depends on the difference between the inner and outer radii, whereas inductance is a function of the 
inner radius. The resonant frequency can be adjusted by changing the inner radius (inductance) or by increasing the difference between the inner and outer radii (capacitance).

In the proposed sensor, a rectangular body replaces the outer periphery as shown in Fig. 1(c). We call this sensor a BLGR. The block shape body provides not only mechanical stability to the LGR, but also a secure mounting place for a coupling loop, which is required to couple RF signals to the LGR. With the BLGR, the resonant frequency can be tuned by adjusting the loop diameter and the gap size (d). The BLGR structure allows cuts perpendicular to the gap. These perpendicular cuts create tightly coupled resonators and thus additional resonant frequencies can be introduced, which is effectively broadens the bandwidth. The cuts go through the inductive loop and the gap. In the case of LGRs used in ESR spectroscopy, the cuts go through the inductor loop only. This is done to allow field modulation to penetrate into the sample and do not create coupled resonators [28].

\section{SECTION III. Principle of Operation of the BLGR Sensor}

Molecules interact with EM fields in several ways. Charges in neutral molecules tend to form a dipole moment. The charges, spins, and dipoles in a sample under test respond to the applied EM wave to form an average field [29]. The total EM field includes the effects of both the applied field and the scattered fields from charge, spin, and current rearrangements [30]. The backscattered field is specific to different atoms and ions, resulting in measurable EM field amplitude and phase variations.

The RF reflection coefficient $\left(S_{11}\right)$ has been used to identify doping concentrations in silicon samples [31]. The measured $S_{11}$ data are mapped into the sample's resistance and capacitance to quantify dopant concentration [32]. The BLGR also measures $S_{11}$ data from ions in a water sample. The BLGR can detect the presence of various ions and their concentrations in terms of a change in reflection coefficient. Ions in water shift the resonant frequencies of the resonator [19]. The frequency shift depends on charge number, concentration, and background of the liquid sample [33]-[34][35]. Water samples with different ions and concentrations of a specific ion (i.e., Pb) exhibit amplitude and phase variations of the $S_{11}$ data. Variation can be observed over the entire frequency range, but it is more pronounced near the resonance frequencies of the BLGR.

In Gramse et al. [32], the authors consider only two dopant ion types, which are relatively immobile in the sample. However, many metal ions may be present in a water sample. Moreover, the mobility of these ions can vary substantially depending on the characteristics of the sample. Hence, the investigation of the reflection coefficient to determine the features for different ions and concentrations of a specific metal ion in a water sample is a much more complex task. Hence, a machine learning algorithm is used to extract features, classify different ions, and quantify different concentrations. Specifically, a support vector machine (SVM) or support vector regression (SVR) algorithm is used to process $S_{11}$ raw data. The SVR model is trained with different concentrations of the target (e.g., $\mathrm{Pb}$ ) ion. As a result, the algorithm learns how both the frequency and phase response components of $S_{11}$ data change for different target ions in a water sample.

\section{SECTION IV. Electrical Circuit Model of the BLGR}

The resonant frequency $\left(\omega_{o}\right)$ of a resonator depends on inductance and capacitance [22]. In the BLGR, the value of both the inductance $(L)$ and the capacitance $(C)$ are directly dependent on the structural 
parameters shown in Fig. 1, including the length $(l)$, the gap width $(w)$, the loop radius $(r)$, the separation between plates $(d)$, and the permittivity $(\epsilon)$ in the gap. These relationships are governed by the following equations:

$$
\begin{gathered}
L=\frac{\mu N^{2} \pi r^{2}}{l} \\
C=\quad \frac{\epsilon \frac{l w}{d}}{\omega_{o}}=\sqrt{\frac{1}{L C}}=\sqrt{\frac{d}{\epsilon \mu \pi N^{2} r^{2} w}} .
\end{gathered}
$$

$(1)(2)(3)$

In this work, we focus on a single loop ( $N=1$ ) BLGR made of aluminum (conductivity of $\left.3.77 \times 10^{7} \mathrm{~S} / \mathrm{m}\right)$. One feature of the BLGR is that the resonant frequency is independent of the length $(l)$ of the device. In metal ion sensing applications, increasing the length provides larger volume of sample for higher sensitivity. The BLGR can be designed according to application-specific requirements by adjusting its various physical dimensions. The resonant frequency is inversely proportional to the loop radius $(r)$. A larger loophole can lower the resonant frequency resulting in low electronic cost to operate the BLGR. In this work, single-gap BLGRs with $1 / 2,3 / 8$, and $1 / 4$ in loop diameter are designed. The frequency responses of the three resonators are shown in Fig. 2 . The physical dimensions of the resonators, measured, calculated, simulated resonant frequencies, and quality factors are summarized in Table $\mathrm{I}$.

TABLE I Dimensions of BLGR With Different Loop Radii, Resonant Frequencies, and $Q$ Values

\begin{tabular}{|l|c|c|l|l|l|c|}
\hline Dimensions $(\mathrm{mm})$ & & & Resonant frequency $(\mathrm{MHz})$ & & & \\
\hline \multicolumn{1}{|c|}{$r$} & $d$ & $w$ & Calculated & Measured & Simulated & $Q$ \\
\hline 3.15 & .45 & 9.6 & 1850 & 1804 & 1829 & 81 \\
\hline 4.80 & .45 & 9.4 & 1227 & 1307 & 1275 & 39 \\
\hline 6.35 & .45 & 9.4 & 928 & 1026 & 1002 & 20 \\
\hline
\end{tabular}
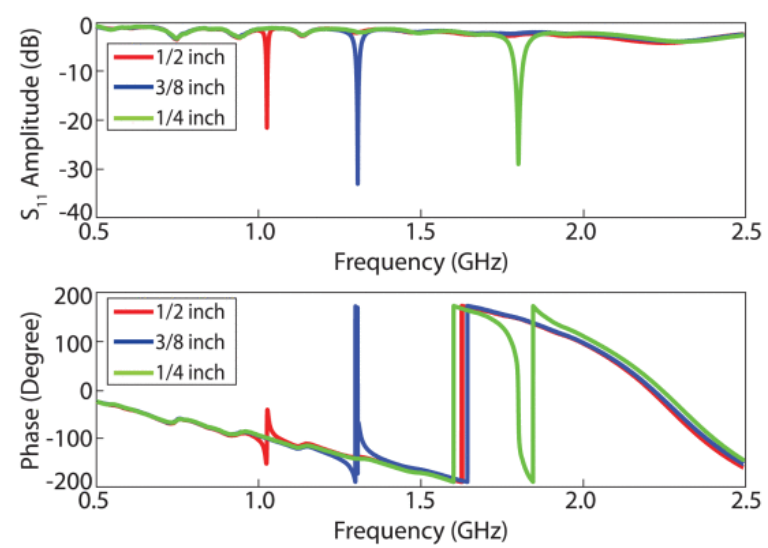

Fig. 2. $S_{11}$ data across the $0.5-2.5 \mathrm{GHz}$ frequency range of single-loop single-gap BLGRs with $1 / 2,3 / 8$, and $1 / 4$ in loop diameter. 
The calculated resonant frequencies in Table I are ideal. Typical deviations from the ideal case include field fringing and losses due to finite conductivity of the metal. The penetration (skin depth) of the electric field into the capacitor plates can also change the capacitance and, hence, the resonant frequency. The measured frequencies in Table I include both effects: fringing and losses. The BLGR geometry is simulated using MIT Electromagnetic Equation Propagation (MEEP) [36] assuming a perfectly conducting material. The simulation results include field fringing effect, but no losses. In Table I, the resonant frequencies from the simulation are within $8 \%$ of the calculated frequencies and within $3 \%$ of the measured results.

In the BLGR, multiple gaps may be made along the length (I) to obtain multiple resonance frequencies for broadening the measurement bandwidth. In that case, the total capacitance is split into several small capacitors/inductors by introducing cuts perpendicular to the original gap as shown in Fig. 3 . The small capacitors/inductors are heavily coupled together and produce multiple resonant frequencies. For instance, a single perpendicular cut divides the total capacitance into two capacitors/inductors. This creates two resonant frequencies, in where the lower resonance is dictated by the total capacitance/inductance and the higher resonant is resulted from the partial capacitance/inductance induced by the additional cut. The lower resonant frequency is slightly higher (2\%) than the resonance frequency with no additional cut. In this work, we use single-gap, double-gap, and triple-gap resonators with a quarter inch loop diameter. Each cut is one-third of the distance (I) from the edge. Fig. 3 shows the geometry of the gap and the additional perpendicular cuts. The cuts are introduced to create tightly coupled resonators that produce extra dips in $S_{11}$. Frequency responses of single-gap, double-gap, and triple-gap resonators are shown in Fig. 4 . The resonant frequency of the single-gap BLGR is $1804 \mathrm{MHz}$ with a $Q$ of 81. The double-gap BLGR has two resonances at 1832 and $2910 \mathrm{MHz}$ with $Q$ values of 20 and 40, respectively. The triple-gap BLGR has three resonances at 1834, 2759, and $3339 \mathrm{MHz}$ with $Q$ values of 20,40 , and 50, respectively. In this work, the $1 / 4^{\prime \prime}$ diameter is chosen because the typical tube size for drinking water system is $1 / 4^{\prime \prime}$.

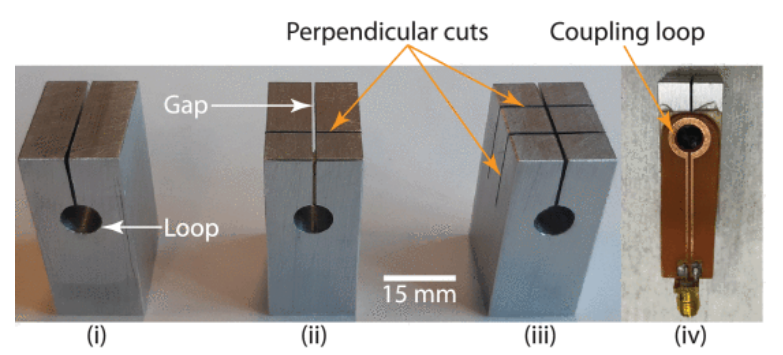

Fig. 3. Single-loop BLGRs with different gaps: 1) single-gap; 2) double-gap; 3) triple-gap; and 4) BLGR attached with an inductive coupling loop. 

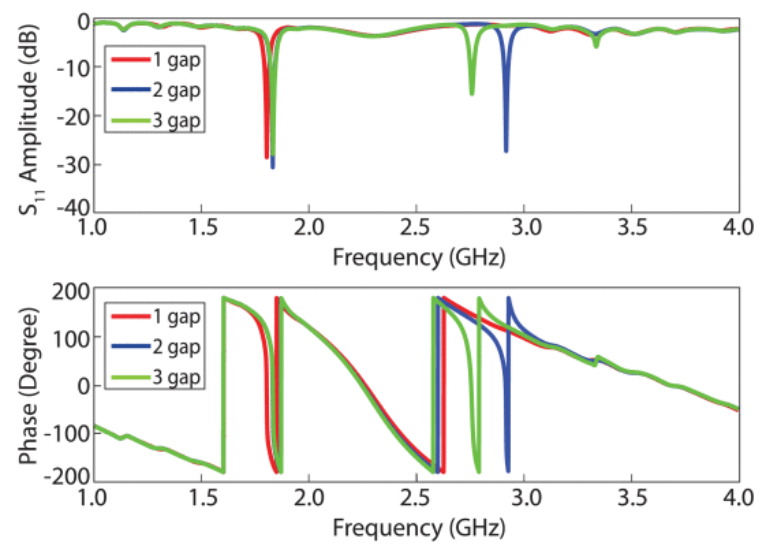

Fig. 4. $S_{11}$ data across the $1-4 \mathrm{GHz}$ frequency range of single-gap, double-gap, and triple-gap BLGR with $1 / 4$ in loop diameter.

\section{SECTION V. Experimental Measurement Details}

The block diagram for $S_{11}$ measurement is shown in Fig. 5 . The coupling loop is connected to a vector network analyzer (VNA) and is placed near the BLGR as shown in Fig. 6(a). The magnetic field of the coupling loop couples to the resonator through the loop hole shown in Fig. 6(a) [25]. The inductive coupling loop is fabricated on a 1.6-mm thick printed circuit board (PCB). The circuit model of the coupling loop and the BLGR is shown in Fig. 6(b). LC and Li are the inductance of the coupling loop and the BLGR, respectively. $\mathrm{Li}$ and $\mathrm{Ci}$ are the inductance and the capacitance of the ith gap in a multiple-gap BLGR. Coupling efficiency depends on the spacing ( $\mathrm{g}$ ) between the coupling loop and the BLGR, respectively. A coupling factor is defined as the ratio of the $S_{11}$ amplitude to the maximum value of the $S_{11}$ at the resonant frequency. The variation of the coupling factor as a function of the distance between the coupling loop and the resonator is shown in Fig. 6(c). The RF energy transferred to the BLGR is maximized when the separation is around $0.9 \mathrm{~mm}$. The resonator reflects a portion of the signal, which is returned to the VNA. The ratio of the reflected signal to the incident signal is the $S_{11}$ scattering parameter. A 40-GHz VNA (Agilent E8363B) is used for $S_{11}$ data acquisition in this work. For practical applications, a Copper Mountain Technology one-port VNA (1 MHz-6 GHz), which is smaller and low cost, can be used.

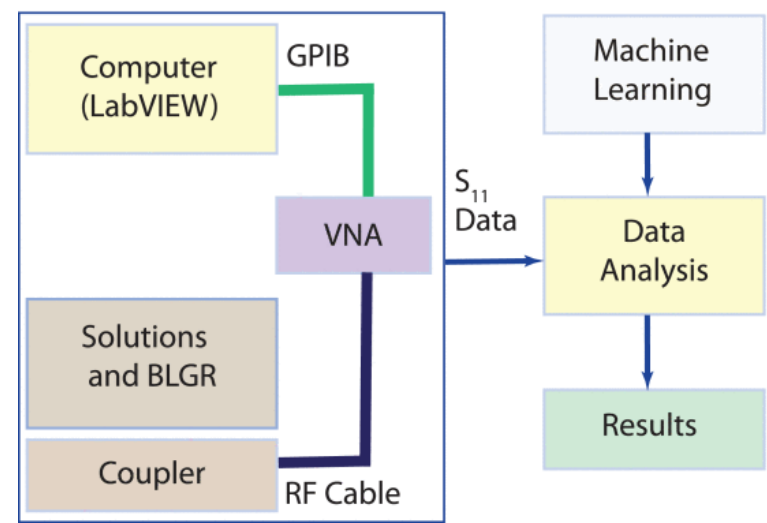

Fig. 5. Block diagram of the proposed strategy for ion sensing in liquid samples using BLGR and machine learning. 


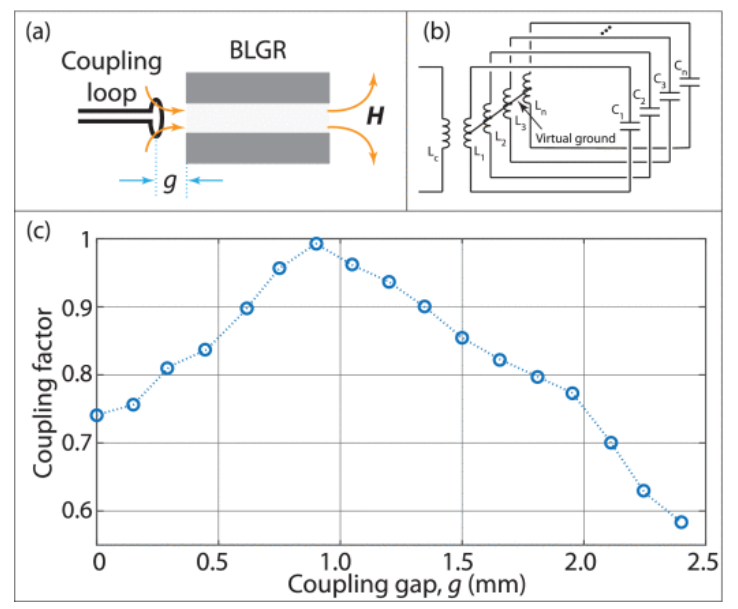

Fig. 6. Inductive loop coupling mechanism of BLGR: (a) orientation of magnetic field, (b) generalized equivalent circuit of multiple gap BLGR, and (c) variation of coupling factor with coupling space, $g$.

A quarter-inch outer diameter (OD) glass tube is inserted through the coupling loop and the loophole of the BLGR. Various liquid samples are placed in the glass tube. The $S_{11}$ data acquisition process is automated by using a custom LabVIEW program. The liquid samples are placed into the glass tube in the BLGR using a syringe pump. An initial delay of $10 \mathrm{~s}$ is used to settle the liquid sample motion before the $S_{11}$ measurement begins. The frequency range of the $S_{11}$ data is from $10 \mathrm{MHz}$ to $5 \mathrm{GHz}$ with 20000 data points. The VNA measures the amplitude and the phase at each frequency. For each measurement, the 20000 magnitude and phase values are normalized to the range [0,1] and concatenated into 40 000-dimensions feature vectors, which are used to train the SVM and the SVR.

\section{SECTION VI. Results}

In this section, two methods for measuring metal ions in water samples using the BLGR are presented: classification of different ions $\left(\mathrm{Na}^{+}, \mathrm{K}^{+}\right.$, and $\left.\mathrm{Mg}^{2+}\right)$ in $\mathrm{DI}$ water and measurement of the concentration of $\mathrm{Pb}$ ions in drinking water.

Many unknown ions dissolved in city water may affect the $S_{11}$ measurement. To verify that our algorithms are able to determine the presence of ions of interest in water samples, $100 \mathrm{ppm} \mathrm{Na}^{+}, \mathrm{K}^{+}$, and $\mathrm{Mg}^{2+}$ solutions are prepared by dissolving sodium chloride $(\mathrm{NaCl})$, potassium chloride $(\mathrm{KCL})$, and magnesium chloride hexahydrate $\left(\mathrm{MgCl}_{2} \cdot 6 \mathrm{H}_{2} \mathrm{O}\right)$ in DI water, respectively. A multiclass classifier based on error-correcting output codes [37] comprising three binary SVM classifiers is trained with 1300 feature vectors from each sample and tested on an additional 200 feature vectors of each sample. Table II shows the confusion matrix corresponding to the 200 test feature vectors for each of the three ions. As the table indicates, each of the 200 predicted feature vectors is associated with the correct ion, that is, the test data is classified with $100 \%$ accuracy.

TABLE II Machine Learning Algorithm Results for $\mathrm{Na}+, \mathrm{K}+$, and Mg2+ Classification Test

\begin{tabular}{|l|l|l|l|l|}
\hline & Predicted & & & \\
\hline & & $\mathrm{Na}^{+}$ & $\mathrm{K}^{+}$ & $\mathrm{Mg}^{2+}$ \\
\hline Actual & $\mathrm{Na}^{+}$ & 200 & 0 & 0 \\
\hline & $\mathrm{K}^{+}$ & 0 & 200 & 0 \\
\hline
\end{tabular}


from left to right along with the training level of each ppb concentration. The $x$-axis represents the sample number and the $\mathrm{y}$-axis represents the $\mathrm{Pb}$ concentration in $\mathrm{ppb}$. As shown in Fig. 7, $\mathrm{Pb}$ measurement using the triple-gap BLGR shows the lowest error.

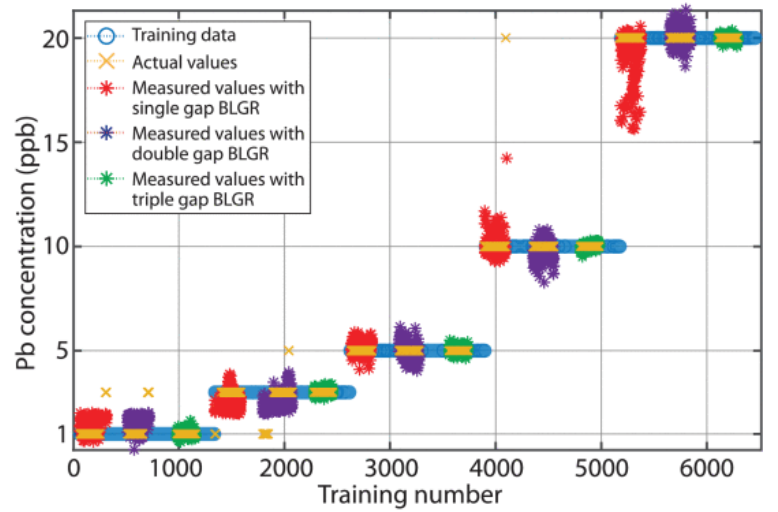

Fig. 7. Machine learning training and test results of $\mathrm{Pb}$ measurement in city water. 1, 3, 5, 10, and $20 \mathrm{ppb} \mathrm{Pb}$ concentrations are measured. Test results of a single-gap, double-gap, and triple-gap BLGR are spaced horizontally from left to right on the training datasets.

The rms errors for each $\mathrm{Pb}$ concentration for the three different resonators are summarized in Table IV. The average rms errors of the measurements are $0.70,0.58$, and $0.16 \mathrm{ppb}$ for a single-gap, doublegap, and triple-gap BLGR, respectively.

TABLE IV RMS Error of Measurement for Different Pb Concentrations and Resonators

\begin{tabular}{|l|l|l|l|}
\hline Concentration & RMS error $(\mathrm{ppb})$ & & \\
\hline$(\mathrm{ppb})$ & Single-gap & Double-gap & Triple-gap \\
\hline 1 & 0.53 & 0.86 & 0.18 \\
\hline 3 & 0.63 & 0.60 & 0.15 \\
\hline 5 & 0.34 & 0.47 & 0.17 \\
\hline 10 & 0.44 & 0.54 & 0.16 \\
\hline 20 & 1.55 & 0.44 & 0.15 \\
\hline
\end{tabular}

\section{A. Interpolation Performance}

Since the SVR is trained using discrete concentration values, it must be able to interpolate samples with previously unobserved concentrations. To evaluate the ability of the model to interpolate concentration values between the trained values (i.e., not observed during training), the performance of each of the resonators is assessed using 200 new samples with a Pb concentration of $15 \mathrm{ppb}$, which is not included in the training sets described above. As Fig. 8 indicates, for the single-gap resonator, the measurement error is within $25 \%$ of the actual value, which corresponds to an rms error of $2.42 \mathrm{ppb}$. The estimates generated using the double-gap resonator differ by at most $13 \%$ from the actual values, which corresponds to an rms error of $0.71 \mathrm{ppb}$. Although the estimates generated by the triple-gap resonator show lower variance than those obtained using the double-gap resonator, the bias of approximately $1 \mathrm{ppb}$ for the triple-gap resonator leads to a higher rms error of $1.68 \mathrm{ppb}$ with respect to the actual values. 


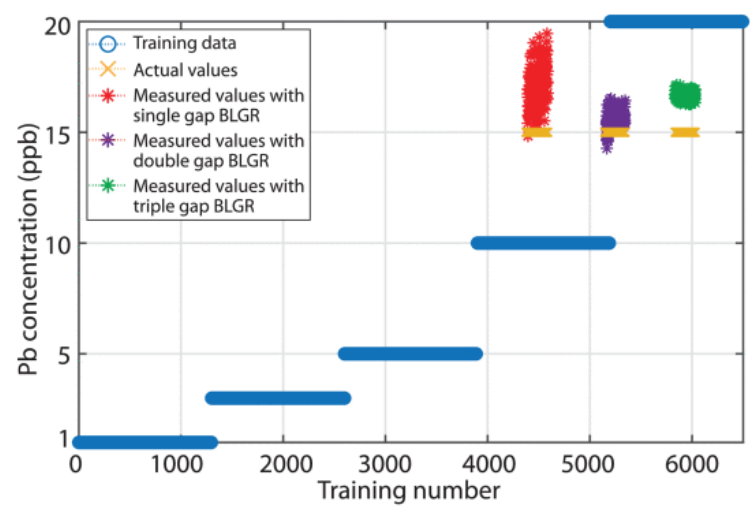

Fig. 8. Performance of the proposed machine learning algorithm in the presence of previously unobserved concentrations. Test results of a single-gap, double-gap, and triple-gap BLGR are spaced horizontally from left to right on the training datasets.

It should be noted that the precision of the interpolated measurements can be adjusted based upon the resolution of the training samples. Let $\mathrm{li}, \mathrm{i}=1,2, \ldots, \mathrm{N}$ represent the $\mathrm{N}$ different concentration levels used for training (in the experiments above, $\mathrm{N}=5$ ). Let $\mathrm{I}$ be an arbitrary concentration level such that $l i-1 \leq \mid \sim \leq i$. The overestimation error for $\mid \sim$ is upper bounded by li and lower bounded by li-1 . Hence, the overall error is limited to li-li-1. For example, for a concentration of $2 \mathrm{ppb}$, the measured values are expected to be between 1 and $3 \mathrm{ppb}$, whereas for a concentration of $15 \mathrm{ppb}$, the corresponding error is at most $10 \mathrm{ppb}$ (i.e., $\mathrm{li}-1=10$ and $\mathrm{li}=20$ ). Therefore, by increasing the resolution of the training data, it is possible to reduce the interpolation error to the desired sensor resolution. For example, training with samples at $1 \mathrm{ppb}$ intervals would allow the sensor to estimate Pb levels with 1 ppb resolution.

\section{B. Robustness to Variation in Water Composition}

To assess the robustness of the proposed approach to daily variations in the composition of water samples, the performance of the machine learning algorithm is evaluated using training data and test data collected on different days. Water samples from the city of Milwaukee are collected every other day on six different days. Pb solutions are diluted into the samples collected in days 3, 5, and 7 at concentrations of 5, 10, and $20 \mathrm{ppb}$. These samples are used to train the SVR. The test set consists of the samples from days 1,9 , and 11 diluted with $\mathrm{Pb}$ at a concentration of $10 \mathrm{ppb}$. The $\mathrm{Pb}$ concentrations of samples in the test set are measured using the triple-gap resonator. Table $V$ shows that although variations in $\mathrm{pH}$, conductivity, and total dissolved solids (TDSs) lead to slightly higher rms errors, the average error is comparable to that obtained using training and test samples collected on the same day. Training the algorithm using data collected over longer periods of time should further mitigate the impact of water composition variability on the performance of the sensor. For example, the model could be trained using samples collected once per week over a year. Since most deviations in water composition should be observable during such an extended period, training with yearly data should further improve the performance of the machine learning algorithm.

TABLE V Summary of pH, Conductivity, and TDS of Water Samples (No Pb) Collected on Different Days. RMS Error (Right Column) of 10 ppb Pb Samples Tested With the SVR Model 


\begin{tabular}{|l|l|l|l|l|l|}
\hline & Day & $\mathrm{pH}$ & $\begin{array}{l}\text { Conduct1V1ty } \\
(\mu \mathrm{S} / \mathrm{cm})\end{array}$ & $\begin{array}{l}\text { TDS } \\
(\mathrm{ppm})\end{array}$ & $\begin{array}{l}\text { RMS error } \\
(\mathrm{ppb})\end{array}$ \\
\hline & 3 & 7.81 & 316.8 & 207.8 & - \\
\hline Training data & 5 & 7.68 & 319.6 & 209.6 & - \\
\hline & 7 & 7.74 & 312.4 & 204.7 & - \\
\hline Average & & 7.74 & 316.3 & 207.4 & - \\
\hline & 1 & 7.87 & 309.0 & 202.3 & 1.45 \\
\hline Test data & 9 & 7.65 & 314.0 & 205.6 & 0.44 \\
\hline & 11 & 7.72 & 312.2 & 203.9 & 0.15 \\
\hline Average & & 7.75 & 311.7 & 203.9 & 0.68 \\
\hline
\end{tabular}

\section{Measurement Accuracy in the Presence of Interfering Metal lons}

$\mathrm{Cu}, \mathrm{Fe}$, and $\mathrm{Zn}$ metal ions and their compounds are frequently found in tap water and typically occur at concentrations that are higher than more regulated heavy metals, such as $\mathrm{Pb}, \mathrm{Cd}, \mathrm{Hg}$, and $\mathrm{As}$. The Environmental Protection Agency (EPA) maximum contaminant levels (MCLs) for $\mathrm{Cu}$, Fe, and $\mathrm{Zn}$ can be one to two orders of magnitude greater than the regulated limit for $\mathrm{Pb}$ and other higher toxicity heavy metals. Interference due to high concentrations of $\mathrm{Cu}, \mathrm{Fe}$, and $\mathrm{Zn}$ on the sensor's response to $\mathrm{Pb}$ is investigated here.

The SVR is trained with 5, 10, and $20 \mathrm{ppb} \mathrm{Pb}$ solutions prepared in Milwaukee city water. First $\mathrm{Cu}, \mathrm{Fe}$, and $\mathrm{Zn}$ solutions of $10 \mathrm{ppb}$ without $\mathrm{Pb}$ ions are tested with the model trained using $\mathrm{Pb}$ ions. The results indicate no $\mathrm{Pb}$ ions in the test samples. Then, $10 \mathrm{ppb} \mathrm{Pb}$ solutions spiked with interfering $\mathrm{Cu}$ ions (20, 50 , and $100 \mathrm{ppb}$ ) are prepared. Table VI shows the resulting rms errors for Pb concentrations when spiked with $\mathrm{Cu}$ ions. The results show that the presence of $\mathrm{Cu}$ ions has no significant impact on $\mathrm{Pb}$ concentration measurement. In each case, the BLGR can effectively estimate $\mathrm{Pb}$ concentrations in water with the background interfering ions. The average rms error is $1.34 \mathrm{ppb}$ over the three interfering ions.

TABLE VI Summary of Measurement Error of $10 \mathrm{ppb}$ Pb Solutions Spiked With Interfering Metal Ions of Different Concentrations

\begin{tabular}{|l|l|l|}
\hline Metal ions & Concentration (ppb) & RMS error (ppb) \\
\hline Cu2 + & 20 & 0.46 \\
\hline & 50 & 3.16 \\
\hline & 100 & 1.15 \\
\hline Fe3 + & 20 & 2.03 \\
\hline & 50 & 2.03 \\
\hline & 100 & 0.83 \\
\hline Zn2 + & 20 & 0.39 \\
\hline & 50 & 0.49 \\
\hline & 100 & 1.58 \\
\hline
\end{tabular}




\section{SECTION VII. Discussion}

In this work, the impact of elements found in drinking water on the accuracy of the results is considered. For example, $\mathrm{HNO}_{3}$ is added to city water to prevent precipitation of $\mathrm{PbCl}_{2}$. Since both $\mathrm{HNO}_{3}$ and $\mathrm{PbCl}_{2}$ are dissolved in the city water, the $\mathrm{BLGR}$ may be detecting ions from $\mathrm{HNO}_{3}$ instead of $\mathrm{Pb}$ ions. $\mathrm{A} 1 \% \mathrm{HNO}_{3}$ seed solution is used as a control solution to confirm that the sensor measures $\mathrm{Pb}$ ions rather than ions from $\mathrm{HNO}_{3}$. Control solutions (with no $\mathrm{Pb}$ ) are prepared assuming equal amounts of $\mathrm{HNO}_{3}$ in the control solution and the $\mathrm{Pb}$ solution for the 10 and $20 \mathrm{ppb}$ levels. The measured $\mathrm{Pb}$ levels of both control solutions are close to $0 \mathrm{ppb}$.

There are other typical contaminants (minerals and other metal ions) in drinking water that may affect our results. Conductivity is related to the total dissolved ion content in the water. DI water has a $\mathrm{pH}$ of 6.07 and a conductivity of $1.65 \mu \mathrm{S} / \mathrm{cm}$. The conductivity of city water $(308.6 \mu \mathrm{S} / \mathrm{cm})$ is very different from that of $\mathrm{DI}$ water. $\mathrm{Pb}$ concentration is measured using city water solutions for machine learning training and DI water solutions in testing and vice versa. The results indicate that the background water chemistry may influence the measurement of $\mathrm{Pb}$ concentration. As the background chemistry changes, additional changes in the machine learning model may be required.

The concentration of dissolved metal ions in drinking water may also affect the results. The concentration of metal ions in the city water samples is determined using inductively coupled plasma (ICP) analysis. The presence of $\mathrm{Na}, \mathrm{Mg}, \mathrm{Al}, \mathrm{K}, \mathrm{Ca}, \mathrm{Fe}, \mathrm{Cu}$, and $\mathrm{Zn}$ ions are found to be between $5 \mathrm{ppb}$ and $35 \mathrm{ppm}$, while concentrations of $\mathrm{Cu}, \mathrm{Fe}$, and $\mathrm{Zn}$ are 23, 20, and $4 \mathrm{ppb}$, respectively. $\mathrm{Pb}$ solutions are additionally spiked with 20,50, and $100 \mathrm{ppb}$ of $\mathrm{Cu}, \mathrm{Fe}$, and $\mathrm{Zn}$ ions, respectively. The rms error of the solutions containing only $\mathrm{Pb}$ is less than $1 \mathrm{ppb}$. The average measurement error of $\mathrm{Pb}$ concentration is up to $13 \%$ for samples intentionally spiked with interfering ions. Training the SVR with samples containing different concentrations of interfering ions commonly found in city water would further reduce the impact of these ions on the accuracy of Pb estimation.

It is also possible to use the measured data to infer an appropriate frequency range to enhance selectivity. For example, using a single-gap BLGR to estimate Pb concentration in DI water, we search for the $1 \mathrm{GHz}$ frequency span within the $10 \mathrm{MHz}$ to $5 \mathrm{GHz}$ range with the smallest rms errors in $\mathrm{Pb}$ concentration. The segment containing resonance has the lowest error (25\% lower than the other segments). Therefore, a multiple gap BLGR may estimate ion concentrations more accurately because there are multiple resonant frequencies. We have measured $\mathrm{Pb}$ concentration in city water using a single-gap, double-gap, and triple-gap BLGR over the entire frequency range ( $10 \mathrm{MHz}$ to $5 \mathrm{GHz}$ ). The average rms errors of each estimate are $0.70,0.58$, and $0.16 \mathrm{ppb}$, respectively. As expected, the triplegap BLGR having three resonant frequencies in the scan frequency range shows the lowest errors. The triple-gap BLGR has three resonant frequencies, each with a different $Q$. The most sensitive resonance for $\mathrm{Pb}$ ions in water is found by computing rms errors in $\mathrm{Pb}$ concentration for a $1 \mathrm{GHz}$ frequency span around each resonant frequency. The extracted rms errors are 0.30, 0.26, and $0.29 \mathrm{ppb}$, for the first, second, and third frequency ranges, respectively. Hence, the second frequency span contains more information about $\mathrm{Pb}$ ion concentration. A BLGR with all three resonances (triple-gap) in the second frequency range $(2.25-3.25 \mathrm{GHz})$ may result in lower errors in $\mathrm{Pb}$ measurement. 
The machine learning algorithms can also be adapted to automatically determine a suitable frequency to further improve sensor selectivity. Feature selection techniques such as those proposed in [38] may be used to allow the machine learning algorithms to automatically determine the most selective resonant frequency for $\mathrm{Pb}$ ions. A preliminary feature analysis indicates that the algorithms take into consideration distinct frequency ranges for determining the presence of different ions. Furthermore, the algorithms extract most of the relevant information from the phase response of the reflected signal. Such behavior is consistent with the fact that the response of the ions to the excitation signal should be better characterized in terms of its delay than its attenuation. The results of a feature selection study can then be used to design a suitable BLGR with optimum center frequency. This approach would predict $\mathrm{Pb}$ levels in water with higher accuracy while simultaneously suppressing the effect of other contaminants.

In terms of data acquisition and processing time, measuring the $S_{11}$ raw signal using the Agilent E8363B VNA over the frequency range described above takes $9.1 \mathrm{~s}$, and the SVR processes the corresponding sensor measurement in $10 \mathrm{~ms}$ on average on an Advanced Micro Devices (AMD) Rome 64-core $2 \mathrm{GHz}$ processor (running on a single core). Hence, the proposed system can perform approximately six measurements per minute. Since the concentration of contaminants in drinking water does not vary significantly within such short intervals, the sensor allows on-site/in situ detection of metal ions continuously and in real-time.

\section{SECTION VIII. Conclusion}

A continuous on-site/in situ sensing system for metal ions in water is presented. There is no physical contact of the water samples with the sensing elements, which increases sensor longevity and retains its functional consistency. The conventional outer circular shape of LGRs is replaced with a rectangular block for mechanical stability, coupling loop support, and multiple cuts for multiple resonant frequencies. The proposed BLGR has a compact size, highly tunable resonant frequencies, moderate $Q$, and flexible integration into existing water supply systems. Machine learning algorithms (SVM and SVR) replace existing mathematical models limited to immobile ions in a solid substrate. Metal ion concentrations in water are estimated using $S_{11}$ raw data alone. The measurement error decreases with an increase in the number of gaps in the BLGR because there are more resonant frequencies. The triple-gap BLGR has the lowest rms error ( $0.16 \mathrm{ppb}$ average error) in $\mathrm{Pb}$ measurement. The detection limit of $1 \mathrm{ppb} \mathrm{Pb}$ is achieved in city water containing various unknown background ions; this is significantly below the United States EPA regulation (15 ppb).

\section{ACKNOWLEDGMENT}

The authors would like to thank Avijit Hira for his work on this investigation and in the preparation of the article; also like to thank A. O. Smith and Pentair for sample preparation and discussions; and also like to thank Tom Silman for machining the BLGRs used in this work.

Water Equipment and Policy (WEP) operates under the auspices of the National Science Foundation Industry/University Cooperative Research Center Program. 


\section{References}

1. Q.-Z. Hu and C.-H. Jang, "Liquid crystal-based sensors for the detection of heavy metals using surface-immobilized urease", Colloids Surf. B Biointerfaces, vol. 88, pp. 622-626, Dec. 2011.

2. K. M. Dean, Y. Qin and A. E. Palmer, "Visualizing metal ions in cells: An overview of analytical techniques approaches and probes", Biochim. Biophys. Acta (BBA)-Mol. Cell Res., vol. 1823, no. 9, pp. 1406-1415, Sep. 2012.

3. C.-S. Wu, M. K. K. Oo and X. Fan, "Highly sensitive multiplexed heavy metal detection using quantum-dot-labeled DNAzymes", ACS Nano, vol. 4, no. 10, pp. 5897-5904, Oct. 2010.

4. A. Montaser, Inductively Coupled Plasma Mass Spectrometry, Hoboken, NJ, USA:Wiley, 1998.

5. H. Wang, Z. Wu, B. Chen, M. He and B. Hu, "Chip-based array magnetic solid phase microextraction on-line coupled with inductively coupled plasma mass spectrometry for the determination of trace heavy metals in cells", Analyst, vol. 140, no. 16, pp. 5619-5626, 2015.

6. B. Welz and M. Sperling, Atomic Absorption Spectrometry, Hoboken, NJ, USA:Wiley, 2008.

7. T. Gong, J. Liu, X. Liu, J. Liu, J. Xiang and Y. Wu, " A sensitive and selective sensing platform based on CdTe QDs in the presence of $L$-cysteine for detection of silver mercury and copper ions in water and various drinks ", Food Chem., vol. 213, pp. 306-312, Dec. 2016.

8. V. K. Gupta, M. R. Ganjali, P. Norouzi, H. Khani, A. Nayak and S. Agarwal, "Electrochemical analysis of some toxic metals by ion-selective electrodes", Crit. Rev. Anal. Chem., vol. 41, no. 4, pp. 282313, 2011.

9. L. Cui, J. Wu and H. Ju, "Electrochemical sensing of heavy metal ions with inorganic organic and biomaterials", Biosensors Bioelectron., vol. 63, pp. 276-286, Jan. 2015.

10. C. A. Parker and W. T. Rees, "Fluorescence spectrometry. A review", Analyst, vol. 87, no. 1031, pp. 83-111, 1962.

11. R. Sitko, P. Janik, B. Zawisza, E. Talik, E. Margui and I. Queralt, "Green approach for ultratrace determination of divalent metal ions and arsenic species using total-reflection X-ray fluorescence spectrometry and mercapto-modified graphene oxide nanosheets as a novel adsorbent", Anal. Chem., vol. 87, no. 6, pp. 3535-3542, Mar. 2015.

12. X. Hou, R. S. Amais, B. T. Jones and G. L. Donati, "Inductively coupled plasma optical emission spectrometry" in Encyclopedia of Analytical Chemistry, Hoboken, NJ, USA:Wiley, 2000.

13. V. N. Losev, O. V. Buyko, A. K. Trofimchuk and O. N. Zuy, "Silica sequentially modified with polyhexamethylene guanidine and Arsenazo I for preconcentration and ICP-OES determination of metals in natural waters", Microchem. J., vol. 123, pp. 84-89, Nov. 2015.

14. B. Bansod, T. Kumar, R. Thakur, S. Rana and I. Singh, "A review on various electrochemical techniques for heavy metal ions detection with different sensing platforms", Biosensors Bioelectron., vol. 94, pp. 443-455, Aug. 2017.

15. B. Çeken, M. Kandaz and A. Koca, "Electrochemical metal-ion sensors based on a novel manganese phthalocyanine complex", Synth. Met., vol. 162, no. 17, pp. 1524-1530, 2012.

16. F. Long, A. Zhu, H. Shi, H. Wang and J. Liu, " Rapid on-site/ in-situ detection of heavy metal ions in environmental water using a structure-switching DNA optical biosensor ", Sci. Rep., vol. 3, no. 1, pp. 1-7, Dec. 2013.

17. W. B. Ji et al., "Detection of low-concentration heavy metal ions using optical microfiber sensor", Sens. Actuators B Chem., vol. 237, pp. 142-149, Dec. 2016.

18. C. G. Juan, E. Bronchalo, B. Potelon, C. Quendo, E. Ávila-Navarro and J. M. Sabater-Navarro, "Concentration measurement of microliter-volume water-glucose solutions using $Q$ factor of microwave sensors", IEEE Trans. Instrum. Meas., vol. 68, no. 7, pp. 2621-2634, Jul. 2019. 
19. K. Zhang, R. K. Amineh, Z. Dong and D. Nadler, "Microwave sensing of water quality", IEEE Access, vol. 7, pp. 69481-69493, 2019.

20. S. Harnsoongnoen, "Metamaterial-inspired microwave sensor for detecting the concentration of mixed phosphate and nitrate in water", IEEE Trans. Instrum. Meas., vol. 70, pp. 1-6, 2021.

21.T . Sphicopoulos and F. Gardiol, "Slotted tube cavity: A compact resonator with empty core", IEE Proc. H-Microw. Antennas Propag., vol. 134, no. 5, pp. 405-410, Oct. 1987.

22. W. N. Hardy and L. A. Whitehead, "Split-ring resonator for use in magnetic resonance from 2002000 MHz", Rev. Sci. Instrum., vol. 52, no. 2, pp. 213-216, Feb. 1981.

23. M. Mehdizadeh, T. K. Ishii, J. S. Hyde and W. Froncisz, "Loop-gap resonator: A lumped mode microwave resonant structure", IEEE Trans. Microw. Theory Techn., vol. MTT-31, no. 12, pp. 1059-1064, Dec. 1983.

24. G. A. Rinard and G. R. Eaton, "Loop-gap resonators" in Biological Magnetic Resonance Biomedical EPR Part B: Methodology Instrumentation and Dynamics, New York, NY, USA:Springer, vol. 24, 2005.

25. W. Froncisz and J. S. Hyde, "The loop-gap resonator: A new microwave lumped circuit ESR sample structure", J. Magn. Reson., vol. 47, no. 3, pp. 515-521, May 1982.

26. D. D. Thomas, C. H. Wendt, W. Francisz and J. S. Hyde, "Saturation transfer EPR spectroscopy on spin-labeled muscle fibers using a loop-gap resonator", Biophys. J., vol. 43, no. 1, pp. 131-135, Jul. 1983.

27. R. L. Wood, W. Froncisz and J. S. Hyde, "The loop-gap resonator. II. Controlled return flux threeloop two-gap microwave resonators for ENDOR and ESR spectroscopy", J. Magn. Reson., vol. 58, no. 2, pp. 243-253, Jun. 1984.

28. Y. Sakamoto, H. Hirata and M. Ono, "Design of a multicoupled loop-gap resonator used for pulsed electron paramagnetic resonance measurements", IEEE Trans. Microw. Theory Techn., vol. 43, no. 8, pp. 1840-1847, Aug. 1995.

29. J. Baker-Jarvis and S. Kim, "The interaction of radio-frequency fields with dielectric materials at macroscopic to mesoscopic scales", J. Res. Nat. Inst. Standards Technol., vol. 117, pp. 1-60, Feb. 2012.

30. J. Baker-Jarvis, P. Kabos and C. L. Holloway, "Nonequilibrium electromagnetics: Local and macroscopic fields and constitutive relationships", Phys. Rev. E Stat. Phys. Plasmas Fluids Relat. Interdiscip. Top., vol. 70, no. 3, Sep. 2004.

31. E. Brinciotti et al., "Probing resistivity and doping concentration of semiconductors at the nanoscale using scanning microwave microscopy", Nanoscale, vol. 7, no. 35, pp. 14715-14722, 2015.

32. G. Gramse, M. Kasper, L. Fumagalli, G. Gomila, P. Hinterdorfer and F. Kienberger, "Calibrated complex impedance and permittivity measurements with scanning microwave microscopy", Nanotechnology, vol. 25, no. 14, Apr. 2014.

33. M. N. Gaboriaud, M. Desaintfuscien and F. G. Major, "Absolute measurement of the total number of ions stored in an RF quadrupole trap", Int. J. Mass Spectrometry lon Phys., vol. 41, no. 1, pp. 109-123, Dec. 1981.

34. A. Rahafrooz and S. Pourkamali, "Detection of sub-ppm traces of aqueous heavy-metal ions using micro-electro-mechanical beam resonators", J. Micromech. Microeng., vol. 19, no. 11, Nov. 2009.

35. C. Ma and A. Wang, "Optical fiber tip acoustic resonator for hydrogen sensing", Opt. Lett., vol. 35, no. 12, pp. 2043-2045, 2010. 
36. A. F. Oskooi, D. Roundy, M. Ibanescu, P. Bermel, J. D. Joannopoulos and S. G. Johnson, "Meep: A flexible free-software package for electromagnetic simulations by the FDTD method", Comput. Phys. Commun., vol. 181, no. 3, pp. 687-702, 2010.

37.S. Escalera, O. Pujol and P. Radeva, "Separability of ternary codes for sparse designs of errorcorrecting output codes", Pattern Recognit. Lett., vol. 30, no. 3, pp. 285-297, 2009.

38.J. M. Valente and S. Maldonado, "SVR-FFS: A novel forward feature selection approach for highfrequency time series forecasting using support vector regression", Expert Syst. Appl., vol. 160, Dec. 2020. 